\title{
Gestión del conocimiento y capacidades de innovación incremental en empresas de México y Bolivia
}

Marco A. Nuñez-Ramírez ${ }^{*}$, Roger A. Banegas-Rivero² ${ }^{2}$ Altayra G. Ozuna-Beltrán ${ }^{3}$ y Jhonny D. Atila-Lijerón ${ }^{4}$ (1) Instituto Tecnológico de Sonora, Departamento de Ciencias Administrativas, 5 de febrero 818 Sur, Ciudad Obregón, Sonora, México (correo-e: marco.nunez@itson.edu.mx).

(2) Universidad Católica Boliviana "San Pablo", Unidad Académica Regional Santa Cruz, Postgrado, Av. Irala No. 373, Santa Cruz de la Sierra, Bolivia. (correo-e: aleconomista@gmail.com).

(3) Instituto Tecnológico de Sonora, Departamento de Contaduría y Finanzas, 5 de febrero 818 Sur, Ciudad Obregón, Sonora, México (correo-e: altayra.ozuna@itson.edu.mx).

(4) Universidad Autónoma Gabriel René Moreno, Facultad de Economía, Av. Busch 244, Santa Cruz de la Sierra, Bolivia (correo-e: jdavidatila@gmail.com).

* Autor a quien debe ser dirigida la correspondencia.

Recibido Feb. 7, 2020; Aceptado Abr. 8, 2020; Versión final Jun. 9, 2020, Publicado Oct. 2020

\section{Resumen}

El objetivo de la presente investigación fue estudiar la relación entre las etapas del proceso de gestión del conocimiento y las capacidades de innovación incremental en 159 empresas de México y Bolivia. La metodología empleada fue cuantitativa con un alcance correlacional y diseño no experimental donde fueron empleados dos cuestionarios con escala Likert de cinco puntos, los cuales fueron validados mediante análisis factorial confirmatorio. Por medio del empleo de correlación y regresión lineal, este estudio muestra que la gestión del conocimiento se asocia de manera significativa y positiva con las capacidades de innovación incremental. Se concluye que la socialización, externalización, combinación e internalización del conocimiento influyen significativamente sobre el desarrollo de las capacidades de innovación incremental.

Palabras clave: gestión del conocimiento; capacidades de innovación incremental; correlación

\section{Knowledge management and incremental innovation capabilities in Mexican and Bolivian firms}

\begin{abstract}
The aim of this research study was to examine the relationship between knowledge management process stages and incremental innovation capabilities in 159 Mexican and Bolivian firms. The methodology was quantitative, correlational in scope, and had a non-experimental design. Two questionnaires with a five-point Likert scale were used, which were validated by confirmatory factor analysis. Using correlation and linear regression, this study showed that knowledge management was significantly and positively associated with incremental innovation capabilities. It is concluded that socialization, externalization, combination, and internalization of knowledge have a significant influence in the development of incremental innovation capabilities.
\end{abstract}




\section{INTRODUCCIÓN}

La innovación ha jugado un rol trascendental dentro del desarrollo de las organizaciones en los últimos años (du Plessis, 2007; Ringberg et al., 2016), lo cual puede ser constatado en el hecho de que el interés por parte de la comunidad científica, así como el número de investigaciones sobre esta variable, ha ido en aumento constante (De Avila et al., 2015). Esto ha permitido el surgimiento de investigaciones tanto empíricas como teóricas. Primeramente, desde la evidencia empírica ha sido posible asociarla con aspectos inherentes a las problemáticas empresariales (Khosravi et al., 2019), en especial, con elementos inmateriales, como es el caso del conocimiento (Nguyen, 2018; Ringberg et al., 2016). Por otro lado, es importante señalar que ésta ha sido estudiada por diferentes aproximaciones teóricas. Una de las propuestas que ha buscado explicar a la innovación es la teoría de recursos y capacidades, la cual, al poner énfasis en la importancia de los activos intangibles, considera a la innovación y al conocimiento como recursos estratégicos que puede ser fuente de ventajas competitivas sostenidas (Barney et al. 2011). De esta manera, es posible observar un fuerte vínculo entre la innovación y la gestión del conocimiento (Khosravi et al., 2019; Scaringella, 2016). Sin embargo, según Darroch y McNaughton (2002), se requiere mayor evidencia empírica para explicar la relación entre dichas variables, en especial, al considerar a los tipos de innovación.

Dentro de las diferentes clasificaciones de esta variable se resalta que la innovación puede ser clasificada de acuerdo a su grado novedad en dos niveles: la innovación radical y la innovación incremental (Souto, 2015). La primera corresponde a una ruptura de lo habitual con el propósito de garantizar un éxito a largo plazo, a través del desarrollo de nuevo conocimiento y tecnología que implique un cambio en los mercados; mientras que la innovación incremental puede ser entendida como una extensión o modificación de los productos o servicios con los que ya cuenta una empresa (du Plessis, 2007). Se resalta que tal taxonomía permite distinguir cómo la innovación puede generar estrategias para las organizaciones (Lundvall et al., 2002).

Para dar continuidad a tales premisas, y siguiendo la teoría de recursos y capacidades (Barney et al., 2011), es necesario considerar que se requiere del desarrollo de ciertas habilidades por parte del recurso humano para llevar a cabo dichos tipos de innovación en las organizaciones a través del fomento de ciertos aspectos que son conocidos como capacidades de innovación (Subramaniam y Youndt, 2005). A continuación, se definen a las variables de estudio -capacidades de innovación incremental y gestión del conocimiento-, para posteriormente formular el planteamiento del problema.

La innovación incremental se basa en el aprovechamiento de los recursos disponibles con el propósito de adaptar los productos o servicios para resolver problemas (Geiger y Finch, 2016), lo cual implica bajos riesgos (Ringberg et al., 2016), y un menor nivel de novedad en contraste con la innovación radical (Souto, 2015). De esta manera, la innovación incremental se encuentra orientada hacia el desarrollo de ciertas habilidades encaminadas hacia la resolución de problemas dentro de la organización. Esto constituye el objetivo esencial de este tipo de innovación; no obstante, para que esto sea posible, se requiere del empleo del conocimiento, lo cual es indispensable para mejorar el desempeño tecnológico de la organización (Scaringella, 2016). Por consecuencia, las capacidades de innovación incremental son las capacidades orientadas a mejorar ciertos productos y/o servicios ya existentes, las cuales difieren de la capacidad de innovación radical que busca transformar dichos aspectos (Subramaniam y Youndt, 2005).

Por otro lado, otra variable intangible que -al igual que la innovación-, ha presentado un aumento de publicaciones en los últimos años de manera considerable es la gestión del conocimiento (Serenko y Dumay, 2015), donde, dentro de las diferentes posturas que han tratado de explicar a esta variable, sobresale la teoría de la creación del conocimiento organizacional (Nonaka et al. 2006), la cual sostiene que la generación y transferencia del conocimiento tácito (no documentado) y explícito (documentado) se lleva a cabo mediante cuatro etapas: socialización, externalización, combinación e internalización del conocimiento (Nonaka y von Krogh, 2009). Asimismo, estos autores expresan que cada una de estas fases se integran dentro de un modelo conocido por el acrónimo SECI, donde cada una de éstas tiene un objetivo diferente.

Primeramente, según Nonaka y von Krogh (2009), dentro de la socialización se busca que el conocimiento tácito, fruto de las experiencias, prácticas y observación, sea compartido con otros individuos a través del diálogo. Posteriormente, la externalización corresponde a la capacidad de interpretar el conocimiento tácito individual en conceptos explícitos y claros, los cuales puedan ser entendibles por los demás miembros de la organización. En cuanto a la combinación, aquí se pretende clasificar las diferentes fuentes de conocimiento explícito. Y, finalmente, está la internalización que busca ser un escenario a través del cual el conocimiento explícito se convierta en tácito mediante la interiorización del mismo por parte de los individuos. Esta es la forma en que se lleva a cabo dicho proceso de manera continua, por lo cual, a este modelo se conoce como espiral, debido a que la conversión del conocimiento debe pasar por las etapas descritas, cambiando de manera constante de conocimiento tácito a explícito y viceversa, y del mismo modo, pasando de la parte individual a la colectiva. Según dichos autores, a través de este proceso es cómo el conocimiento es creado y utilizado dentro de las empresas. 


\section{OTROS ANTECEDENTES}

Nonaka et al. (2006) expresan que la teoría de la creación del conocimiento ha sido empleada para dar soporte teórico a diferentes variables intangibles como es el caso de la innovación. No obstante, desde un punto de vista empírico, la relación entre ambas variables depende en gran medida de las características propias de cada una de las etapas del proceso de generación y transferencia del conocimiento, así como el tipo de innovación. De hecho, se han realizado investigaciones que han medido la relación entre variables asociadas con el conocimiento y la innovación dentro de las organizaciones donde existen posturas encontradas que, en conjunto, muestran la necesidad de mayor evidencia empírica.

Por un lado, para Akram et al. (2011), la innovación es entendida como un resultado de todo el proceso de gestión del conocimiento, en especial, al convertir el conocimiento tácito a explícito. Mientras que, por el otro lado, existe evidencia que sostiene que la relación de la innovación se lleva a cabo solo con ciertas etapas del proceso. En este sentido, Ode y Ayavoo (2019) encontraron que prácticas de la gestión del conocimiento, tales como la generación, el almacenamiento, difusión y aplicación del conocimiento se asocian de manera significativa y positiva con la innovación en empresas de Nigeria. Por otro lado, Walecka-Jankowska (2015), al estudiar a 105 compañías de Polonia, y teniendo como objetivo el evaluar cuáles etapas del proceso de gestión del conocimiento influyen sobre la innovación (la adquisición y desarrollo, la codificación, transferencia y utilización del conocimiento), descubrió que solo las prácticas de adquisición y desarrollo afectan de manera relevante al desarrollo de la innovación organizacional. Asimismo, Cerne et al. (2013), quienes estudiaron a empresas de Eslovenia, España y Corea del Sur, encontraron que el intercambio de conocimiento se asocia de manera significativa con la administración de la innovación, lo cual se hace más patente al considerar el tamaño de la organización.

Si bien la gestión del conocimiento se ha convertido en un elemento determinante para el desarrollo de la innovación (Walecka-Jankowska, 2015) y, considerando que la evidencia que asocia a estas variables es poca (Darroch y McNaughton, 2002), todavía no está del todo claro qué parte o etapa del proceso de gestión del conocimiento favorece de mejor manera a la generación de capacidades de innovación, en especial, a la incremental, tomando en cuenta que esta forma de innovación está presente en todo tipo de empresas (Ringberg et al., 2016). En este sentido, Chang y Lee (2008) encontraron con una muestra de empresas industriales de Taiwán que la capacidad para obtener conocimiento afecta de manera positiva a la innovación tanto técnica como administrativa. Mardani et al. (2018), además de encontrar que la gestión del conocimiento se asocia con la innovación, subrayan que la creación, la integración y aplicación del conocimiento tienen un impacto positivo sobre la innovación en empresas de Irán. Mientras que los hallazgos de Nguyen (2018) muestran que el flujo de conocimiento impacta favorablemente sobre la innovación incremental al estudiar las mencionadas variables en empresas de Vietnam. Por otro lado, Darroch y McNaughton (2002) no ubicaron suficiente evidencia empírica que soporte que la adquisición y diseminación de conocimiento afecten significativamente a la innovación incremental al estudiar una muestra de empresas de Nueva Zelanda. Finalmente, Si la innovación puede ser vista como un resultado del proceso de la gestión del conocimiento (Akram et al., 2011; Quintane et al., 2011), entonces se requiere poner mayor énfasis en cómo la gestión del conocimiento puede favorecer al desarrollo de capacidades que favorezcan a la innovación incremental.

Pero ¿por qué se realizó este estudio en dos países? Para atender a esta cuestión se plantean los siguientes argumentos. Primeramente, como se observa dentro de la evidencia empírica consultada, los estudios que trascienden lo organizacional son pocos. En este caso, solo Cerne et al. (2013) estudia la relación de dichas variables en diferentes países. Por esta razón se pensó realizar el estudio en dos países, aunque el diferenciar a ambos no forma parte del objetivo del mismo. ¿Por qué en países latinoamericanos, en especial, Bolivia y México? Aquí, aunque se trata de una cuestión empírica, se toma como punto de partida la parte teórica. Si bien, tal como se describió en párrafos anteriores, la presente investigación parte de la Teoría de recursos y capacidades (Barney et al., 2011), que entiende al conocimiento e innovación como recursos intangibles de las organizaciones, también se desea considerar al elemento extrínseco de la organizacional. La bibliometría sobre innovación realizada por De Avila et al. (2015) muestra que lo social juega un papel importante para la comprensión de la innovación. Una perspectiva que pone énfasis en esto, es la teoría de los sistemas nacionales de innovación. Esta postura es una visión sistémica, al considerar diferentes factores -sociales, económicos, organizacionales y técnicos-, favorece la generación y transferencia de tecnología (Freeman, 1995), en especial, facilita el desarrollo de activos intangibles en las diferentes regiones (Lundvall et al., 2002). En este mismo sentido, la innovación incremental puede asociarse de manera relevante con el desarrollo local (Barragán y Ayaviri, 2017).

Bajo esta perspectiva, Freeman (1995) expone que, durante los años 80, Latinoamérica presentaban debilidades de los sistemas nacionales de innovación, en específico, respecto al sistema educativo, aprendizaje, investigación y desarrollo, infraestructura para ciencia y tecnología, así como telecomunicaciones. Sin embargo, esto no ha cambiado del todo actualmente. Los países de esta región no sobresalen en el desarrollo de la innovación. Esto mismo se observa dentro del entorno empresarial (Global 
Entrepreneurship Monitor [GEM], 2010), lo cual puede ser entendido desde un punto de vista cultural, pues esta región se ha distinguido por la falta de valores orientados hacia el fomento de la innovación y creatividad dentro de las organizaciones, en parte, debido a que éstas se han caracterizado por ser rígidas y jerárquicas (Hofstede y Hofstede, 2005), situación que comparten tanto México como Bolivia. Asimismo, según el Banco Interamericano para el Desarrollo ([BID], 2010), el fomento del conocimiento es bajo en esta región. Además, a pesar de la importancia que han adquirido el estudio de la innovación y la gestión del conocimiento a nivel global, en estos países todavía queda mucho por avanzar. Mientras que, desde la parte académica, han sido pocas las publicaciones latinoamericanas sobre gestión del conocimiento en revistas de alto impacto (Serenko y Dumay, 2015). Es así que, desde lo teórico como lo empírico, la gestión del conocimiento, entendida como un proceso (Nonaka y von Krogh, 2009), puede concertar con lo planteado por teoría de los sistemas nacionales (Freeman, 1995), pues ambos buscan crear y transferir conocimiento, donde el contexto juega un papel relevante. Entonces, el tener dos muestras de empresas de dos países de la misma región puede aportar evidencia a la presente discusión.

Por otro lado, considerando que la innovación radical ha obtenido mayor interés Tellis et al. (2009), la evidencia empírica acerca de la relación de las variables de estudio-gestión del conocimiento y capacidades de innovación incremental- es escasa y, al mismo tiempo, el presente estudio puede ser una propuesta emergente dentro del contexto latinoamericano, por lo tanto, surgen las siguientes preguntas de investigación: ¿existe relación entre la gestión del conocimiento y las capacidades de innovación incremental en empresas de México y Bolivia? Si es así, ¿cómo se lleva cabo tal relación? Además, retomando que la gestión del conocimiento puede ser considerado como una variable predictora de la innovación (Quintane et al., 2011; Walecka-Jankowska 2015), se plantea otra interrogante: ¿cómo influye la gestión del conocimiento sobre las capacidades de innovación incremental?

Con el propósito de dar respuesta a estas cuestiones, se plantean las siguientes hipótesis de estudio: $\mathrm{H}_{1}$ ) La gestión del conocimiento se asocia de manera positiva y significativa con las capacidades de innovación incremental en empresas de México y Bolivia; $\mathrm{H}_{2}$ ) La gestión del conocimiento tiene un efecto positivo y significativo sobre las capacidades de innovación incremental en empresas de México y Bolivia. Lo anterior es puesto a prueba estadísticamente a través del diseño metodológico que se muestra a continuación.

\section{MATERIALES Y MÉTODOS}

Esta investigación fue de tipo cuantitativa con alcance correlacional. Asimismo, se empleó un corte transversal con un diseño no experimental. Dentro de esta sección se describen los sujetos que formaron parte del estudio, así como los instrumentos cuantitativos de medición. Posteriormente, se muestra el procedimiento que fue llevado a cabo.

\section{Sujetos}

Se realizó un muestreo no probabilístico por conveniencia conformado por 159 empresas del estado de Sonora, en México $(n=99)$ y del Departamento de Santa Cruz, Bolivia $(n=60)$. La muestra estuvo caracterizada por representantes de diferentes empresas, cuyos giros son los siguientes: financiero (15\%), manufactura $(11 \%)$, agroidustrial $(6.3 \%)$, alimentos $(6.3 \%)$, tecnológicas $(4 \%)$, petroquímicas $(1.3 \%)$ y otras (56.1\%).

\section{Instrumentos de medición}

Con el fin de poner a prueba las hipótesis de estudio, se emplearon dos instrumentos de medición. Para la gestión del conocimiento se administró una adaptación al español del cuestionario propuesto por Mihi, García y Rojas (2011), el cual mide las cuatro etapas del proceso de gestión del conocimiento mediante 11 ítems en escala Likert-5 con los siguientes valores: 1 (Totalmente en desacuerdo), 2 (en desacuerdo), 3 (Ni de acuerdo ni en desacuerdo), 4 (De acuerdo) y 5 (Totalmente de acuerdo). Este instrumento mide las cuatro etapas del modelo SECI: socialización (3 ítems), externalización (3 ítems), combinación (3 ítems) e internalización del conocimiento ( 2 ítems). En cuanto a la validez de constructo de este instrumento, los autores citados reportan niveles favorables de validez de constructo al emplear modelación de Ecuaciones Estructurales. Dentro del presente estudio, se empleó análisis factorial exploratorio por medio del análisis factorial de componentes principales con rotación Varimax, a través del cual se encontraron los siguientes valores: prueba KaiserMeyer-Olkin $(\mathrm{KMO}=.863)$ y Esfericidad de Bartlett $\left(\mathrm{X}^{2}=601.39 ; g l=55 ; p<0.001\right)$, donde todos los ítems se agruparon de acuerdo a las cuatro etapas del modelo SECl y, al mismo tiempo, explicaron el $70 \%$ de la varianza total. Dichos resultados reflejan niveles favorables de validez. Además, respecto a la confiabilidad, se obtuvo un valor alpha de .855 para esta variable, lo cual refleja una aceptable consistencia interna. Por otro lado, en cuanto a la capacidad de innovación incremental, se administró una versión en español del cuestionario empleado por Subramaniam y Youndt (2005), compuesto por tres ítems en escala Likert de cinco puntos: 1 (Totalmente en desacuerdo), 2 (en desacuerdo), 3 (Ni de acuerdo ni en desacuerdo), 4 (De acuerdo) 
y 5 (Totalmente de acuerdo). Respecto al presente estudio, esta variable unidimensional reflejó valores favorables en la medición de la validez de constructo $\left(\mathrm{KMO}=.752\right.$; Esfericidad de Bartlett, $\mathrm{X}^{2}=283.72 ; \mathrm{gl}=$ 3; $p<0.001)$, donde un solo factor fue suficiente para explicar una varianza total de $82.9 \%$ mediante el análisis factorial exploratorio. Del mismo modo, la confiabilidad fue aceptable $(\alpha=.896)$.

\section{Procedimiento}

Esta investigación fue planeada y ejecutada por investigadores del Instituto Tecnológico de Sonora, México, de la Universidad Católica Boliviana y la Universidad Autónoma Gabriel René Moreno, Bolivia. En un primer momento, los cuestionarios fueron traducidos del inglés al español y, posteriormente, revisados en cuanto a su contenido y lógica. Asimismo, se solicitó a tres profesores mexicanos que lo validaran en cuanto a su contenido. De esta manera se aplicó una prueba piloto a una pequeña muestra de estudiantes de maestría del Instituto Tecnológico de Sonora, quienes hicieron recomendaciones de mejora para la comprensión y contestación del mismo. Lo mismo se realizó con estudiantes de posgrado de la Universidad Autónoma Gabriel René Moreno de Bolivia. Es importante señalar que los participantes de la prueba piloto se encontraban laborando.

Después de lo anterior, los instrumentos de medición fueron autoadministrados por representantes de las empresas de estudio obteniendo una muestra cercana a los 200 cuestionarios; sin embargo, debido a que algunos de estos estaban incompletos, se obtuvo una muestra final compuesta por 159 empresas de ambos países. La recolección de datos fue realizada mediante tres estrategias: a) algunas empresas fueron visitadas, en especial, donde había convenios de colaboración; b) se invitó a participar a trabajadores que se encontraban en algún evento académico y/o de capacitación dentro de las instalaciones de las universidades mencionadas anteriormente; c) a manera de bola de nieve, algunos encuestados hicieron participes del estudio a otros.

La caracterización de los participantes es la siguiente: puestos gerenciales (13.2\%), jefatura (16.4\%), supervisión (14.5\%), administrativo (22\%), Auxiliar (15.7\%), Operativo (10.7\%) y otro (7.5\%). Luego de ser validados los cuestionarios mediante los análisis factoriales, y con el fin de probar empíricamente las hipótesis planteadas para la presente investigación, fueron empleadas la correlación y regresión lineal. En cuanto a esta última prueba, con el fin de cumplir con sus supuestos, se verificó la correlación de las variables y, del mismo modo, se llevó a cabo un análisis de la colinealidad con el propósito de evitar que la relación entre las variables independientes -las etapas del proceso de gestión del conocimiento- pudiera afectar negativamente a la regresión. Los hallazgos son presentados en la siguiente sección.

\section{RESULTADOS}

Al tratar de probar $\mathrm{H}_{1}$, los hallazgos reflejan que todas las etapas del modelo $\mathrm{SECl}$ de manera individual se asocian de manera positiva y significativa con las capacidades de innovación incremental $(p<0.01)$. De esta manera la primera hipótesis es comprobada empíricamente mediante la correlación con resultados bilaterales (Ver tabla 1). Esto refleja que, cuando alguna etapa de la gestión del conocimiento aumenta, sucede lo mismo respecto a las capacidades de innovación incremental. Por otro lado, al realizar regresión lineal, no se encontraron problemas de multicolinealidad entre las variables independientes, debido a que según Field (2009), las correlaciones tuvieron valores menores a .80. Además, los valores de VIF fueron aceptables en todos los casos debido a que los resultados en este rubro son menores de 10. En la Tabla 2, se muestran 4 modelos que muestran los hallazgos obtenidos de cada una de las etapas del proceso: socialización, externalización, combinación e internalización del conocimiento propuestos por el modelo SECI de la creación del conocimiento. Además, todas las etapas del proceso de gestión del conocimiento afectan de manera positiva y significativa sobre las capacidades de innovación incremental $(p<0.001)$, con explicaciones del modelo $\left(R^{2}\right)$ que fueron más altas para las dos últimas etapas que propone la teoría de la creación del conocimiento: combinación e internalización del conocimiento. De hecho, es importante señalar que esta última estuvo cerca de alcanzar un nivel de explicación cercana al $40 \%$ en cuanto al modelo 4 . Consecuentemente, la presente evidencia soporta empíricamente la segunda hipótesis planteada con empresas de México y Bolivia.

Tabla 1: Correlación de las variables

\begin{tabular}{|l|c|c|c|c|c|}
\hline \multicolumn{1}{|c|}{ Variables } & 1 & \multicolumn{1}{c|}{2} & 3 & 4 & 5 \\
\hline 1. Socialización del conocimiento & 1 & & & & \\
\hline 2. Externalización del conocimiento & $.439^{* *}$ & 1 & & & \\
\hline 3. Combinación del conocimiento & $.292^{* *}$ & $.616^{* *}$ & 1 & & \\
\hline 4. Internalización del conocimiento & $.474^{* *}$ & $.552^{* *}$ & $.547^{* *}$ & 1 & \\
\hline 5. Capacidades de innovación incremental & $.425^{* *}$ & $.399^{* *}$ & $.432^{* *}$ & $.590^{* *}$ & 1 \\
\hline
\end{tabular}


Tabla 2: Coeficientes de regresión sobre las capacidades de innovación incremental

\begin{tabular}{|c|c|c|c|c|}
\hline Variables & Modelo 1 & Modelo 2 & Modelo 3 & Modelo 4 \\
\hline $\begin{array}{l}\text { Socialización del } \\
\text { conocimiento }\end{array}$ & $\begin{array}{l}0.425^{\star \star \star} \\
(.085)\end{array}$ & $\begin{array}{l}.310^{* * *} \\
(.094)\end{array}$ & $\begin{array}{l}.302^{\star \star \star} \\
(.091)\end{array}$ & $\begin{array}{l}.184^{*} \\
(.094)\end{array}$ \\
\hline $\begin{array}{l}\text { Externalización } \\
\text { del conocimiento }\end{array}$ & & $\begin{array}{l}.263^{* *} \\
(.074)\end{array}$ & $\begin{array}{l}.088^{* \star *} \\
(.087)\end{array}$ & $\begin{array}{l}-.011 \\
(.083)\end{array}$ \\
\hline $\begin{array}{l}\text { Combinación del } \\
\text { conocimiento }\end{array}$ & & & $\begin{array}{l}.290^{\star *} \\
(.081)\end{array}$ & $\begin{array}{l}.152^{*} \\
(.080)\end{array}$ \\
\hline $\begin{array}{l}\text { Internalización del } \\
\text { conocimiento }\end{array}$ & & & & $\begin{array}{l}.426^{\star * \star} \\
(.77)\end{array}$ \\
\hline $\mathrm{R}^{2}$ & .181 & .237 & .289 & .376 \\
\hline $\mathrm{R}^{2}$ ajustada & .176 & .227 & .275 & .391 \\
\hline$F$ & $34.66^{* * *}$ & $24.188^{* * *}$ & $21.00^{* * *}$ & $24.752^{* * *}$ \\
\hline VIF & 1.00 & 1.23 & 1.62 & 1.77 \\
\hline
\end{tabular}

\section{DISCUSIÓN}

Dentro de la presente investigación se plantearon tres interrogantes: ¿existe relación entre la gestión del conocimiento y las capacidades de innovación incremental en empresas de México y Bolivia? Si es así, ¿cómo se lleva cabo tal relación? Y, finalmente, ¿cómo influye la gestión del conocimiento sobre las capacidades de innovación incremental? A grandes rasgos, a través de este estudio fue posible responder a estas cuestiones. Es importante considerar que el fomento de las capacidades de innovación incremental juega un relevante dentro del entorno empresarial debido a diferentes aspectos. Primeramente, la innovación incremental está presente en todo tipo de empresa (Ringberg et al., 2016), de ahí la importancia de generar tales capacidades dentro del capital humano. Éstas, al orientarse hacia el desarrollo de alternativas para la resolución de problemas, del mismo modo favorecen de manera significativa el desarrollo tecnológico, a pesar de que su nivel de novedad es menor a la radical (Souto, 2015), y también benefician de manera significativa al desarrollo tecnológico (Scaringella, 2016); no obstante, a pesar de esto, la innovación radical ha tenido un mayor interés (Tellis et al. 2009). Dado que esta variable es un tema emergente dentro del estudio de las organizaciones, surge la necesidad imperante de reflexionar sobre las áreas de oportunidad que pueden surgir en el estudio de las capacidades de innovación incremental dentro de diferentes corpus teóricos y contextos.

El primer vínculo que existe entre la gestión del conocimiento y la innovación, es que ambas variables son activos intangibles que, según la teoría de recursos y capacidades, benefician a las organizaciones (Barney et al., 2011); pero que también, según la teoría de los sistemas nacionales de innovación, son cruciales para el desarrollo de las naciones y la sociedad (Freeman, 1995; Lundvall et al, 2002). Tomando como referencia las etapas del modelo SECl propuesto por la teoría de la creación del conocimiento (Nonaka et al., 2006; Nonaka y von Krogh, 2009), todavía no está del todo claro qué etapa favorece de mejor manera al desarrollo de la innovación, en especial a las capacidades de innovación incremental. Los hallazgos presentados dentro de este estudio reflejan que cada una de las cuatro etapas del modelo -socialización, externalización, combinación e internalización del conocimiento-, se asocian de manera significativa con el desarrollo de las capacidades de innovación incremental. Esto muestra que a mayor desarrollo de las etapas del modelo SECI, también las capacidades de innovación incremental tendrán un aumento favorable. Resultados similares fueron presentados por Ode y Ayavoo (2019), para quienes la generación, el almacenamiento, difusión y aplicación del conocimiento se asocian de manera particular en una forma significativa y positiva con la innovación. A diferencia de lo reportado por Walecka-Jankowska (2015), quien encontró que solo la adquisición y desarrollo afectan a la innovación.

A partir de lo anterior, es posible sostener que cada una de las etapas del modelo SECI impactan de modo individual al desarrollo de las capacidades de innovación incremental. Es decir, dentro del proceso de creación, transferencia e internalización del conocimiento tácito e individual a explícito y colectivo, se estará favoreciendo al fomento de los elementos que intervienen dentro de la innovación incremental, lo cual, a su vez, se verá reflejando en mejoras dentro de los productos y servicios que oferta la organización. Aun cuando existen posturas contrapuestas, debido a que autores como Darroch y McNaughton (2002), no encontraron evidencia que asocie a dichas variables, a través del presente estudio se pudo corroborar que todas las etapas de manera individual impactan de forma positiva en el desarrollo de las capacidades de innovación incremental. Dichos hallazgos se asemejan a los resultados obtenidos por Nguyen (2018). Si bien para Akram et al. (2011), el modelo, considerado como un todo, impacta en la innovación, aquí se encontró que las etapas de manera independiente afectan sobre las capacidades de innovación incremental. 
Considerando que la evidencia empírica citada presenta estudios realizados en empresas de otras regiones del mundo como Asia (Chang y Lee, 2008; Nguyen, 2018), Oceanía (Darroch y McNaughton, 2002), África (Ode y Ayavoo, 2019), y Europa (Cerne et al., 2013; Walecka-Jankowska, 2015), se resalta la imperativa necesidad de tratar de explicar la relación entre estas variables dentro del contexto latinoamericano. Cabe señalar que esta región ha mostrado problemas para destacar a nivel global en cuanto a la innovación dentro del sector empresarial (GEM, 2018). Entonces, si se visualiza a esta variable -innovación- como un producto o resultado de la gestión del conocimiento (Akram et al. 2011; Quintane et al., 2011), se requiere mejorar los procedimientos que fomenten la creación y difusión del conocimiento dentro de las organizaciones latinas. Asimismo, por medio de una muestra de empresas de México y Bolivia fue posible observar que todas las etapas del proceso de gestión del conocimiento juegan un papel crucial para fomentar las capacidades de innovación incremental, lo cual concierne a todo tipo de empresas y, retomando que se carece de innovación radical en esta zona, se debería dar mayor énfasis a la innovación incremental, la cual permite resolver problemas (Geiger y Finch, 2016) y mejorar el desempeño (Scaringella, 2016).

Cabe señalar que, desde un enfoque estadístico, los cuatro modelos de la regresión empleada mostraron resultados significativos y que el nivel de explicación fue en aumento, específicamente en las dos últimas etapas. Sin embargo, en cuanto a la internalización del conocimiento -etapa final del modelo SECl-, donde se supone que el conocimiento se ha convertido en explícito, y puede ser administrado y convertido en mejoras para la organización, el nivel de significancia es menor $(p<0.05)$. Es así que se requiere mayor evidencia empírica con muestras más representativas para poder verificar dichos hallazgos empíricos, los cuales, como ya se ha mencionado anteriormente, vienen a aportar evidencia que permita mejorar la comprensión de la relación existente entre las variables en cuestión, y cómo éstas puede favorecer al contexto latinoamericano, en especial a países como México y Bolivia.

Dentro de las principales limitaciones del estudio destaca el tipo muestreo empleado, al cual es posible añadir el tamaño de la muestra y el tipo de participantes. En cuanto al último aspecto, éste es una limitante debido a que, para algunos autores, cuando se estudian a variables organizaciones, se recomienda encuestar a mandos medios y/o directivos que, en el caso de este estudio, solo representaron el $44.3 \%$ de la muestra. Sin embargo, a pesar de lo señalado, se hace hincapié en que ambas hipótesis planteadas fueron soportadas empíricamente. Asimismo, aunque los resultados no pueden ser del todo generalizados debido al tipo de muestreo empleado -no probabilístico-, esta investigación presenta un bosquejo de las áreas de oportunidad que pueden surgir dentro de una paulatina apertura hacia el desarrollo de activos intangibles, tales como la innovación y el conocimiento, las cuales pueden generar ventajas a largo plazo en una región geográfica donde, según Hofstede y Hofstede (2005), las organizaciones se caracterizan por ser rígidas y jerárquicas.

\section{CONCLUSIONES}

Atendiendo al objetivo y las interrogantes de la presente investigación, este trabajo converge las teorías de recursos y capacidades, la teoría de la creación del conocimiento y la teoría de los sistemas nacionales de innovación con el propósito de atender un vacío teórico y empírico, cuyas conclusiones se presentan a continuación: 1) esta investigación permite comprender la relación entre las etapas del proceso de gestión del conocimiento y las capacidades de innovación incremental en empresas de México y Bolivia; 2) aunque no existe un consenso sobre la relación entre estas variables, el presente estudio encontró que todas las etapas del proceso de gestión del conocimiento -de manera independiente- influyen de manera importante sobre las capacidades de innovación incremental; y 3) estos hallazgos aportan evidencia para explicar el desarrollo de las capacidades de innovación puede ser posible a través de las diferentes etapas de la gestión del conocimiento, el cual ha sido un tema poco estudiado en Latinoamérica.

\section{AGRADECIMIENTOS}

Se gradece el apoyo institucional recibido por parte del Instituto Tecnológico de Sonora a través del Programa de Fomento a la Investigación (PROFAPI) en sus ediciones 2019 y 2020; asimismo, al personal docente y administrativo de la Universidad Católica Boliviana "San Pablo" y de la Universidad Autónoma Gabriel René Moreno, las cuales brindaron diferentes espacios para este proyecto.

\section{REFERENCIAS}

Akram, K., Hafeez, S., y otros tres autores, Role of Knowledge Management to Bring Innovation: An Integrated Approach, International Bulletin of Business Administration, (11), 121-134 (2011).

Banco Interamericano para el Desarrollo. Science, Technology, and Innovation in Latin America and the Caribbean. A Statistical Compendium of Indicators. Nueva York: Inter-American Development Bank (2010).

Barragán, M.C., y Ayaviri, V.D., Innovación y Emprendimiento, y su relación con el Desarrollo Local del Pueblo de Salinas de Guaranda, Provincia Bolívar, Ecuador, http://dx.doi.org/10.4067/S0718-07642017000600009, Información tecnológica, 28(6), 71-80 (2017).

Barney, J.B., Ketchen, D.J., y Wright, M., The Future of Resource-Based Theory: Revitalization or Decline? https://doi.org/10.1177/0149206310391805, Journal of Management, 37(5), 1299-1315 (2011). 
Černe, M., Jaklič, M., y Škerlavaj, M., Management Innovation in Focus: The Role of Knowledge Exchange, Organizational Size, and IT System Development and Utilization, https://doi.org/10.1111/emre.12013, European Management Review, 10(3), 153-166 (2013).

Chang, S., y Lee, M., The Linkage between Knowledge Accumulation Capability and Organizational Innovation, https://doi.org/10.1108/13673270810852359, Journal of Knowledge Management, 12(1), 3-20 (2008).

Darroch, J., y McNaughton, R., Examining the Link between Knowledge Management Practices and Types of Innovation, https://doi.org/10.1108/14691930210435570, Journal of Intellectual Capital, 3(3), 210-222 (2002).

De Avila, J.H., Zawislak, P.A., y otros dos autores, Searching for a Path: A Bibliometric Study on Innovation and Technological Capabilities, https://doi.org/10.5585/iji.v3i2.58, International Journal of Innovation, 3(2), 54-66 (2015).

Du Plessis, M., The Role of Knowledge Management in Innovation, https://doi.org/10.1108/13673270710762684, Journal of Knowledge Management, 11(4), 20-29 (2007).

Field, A., Discovering Statistics, 3를. Ed., Londres: Inglaterra (2009).

Freeman, C., The 'National System of Innovation' in Historical Perspective, https://doi.org/10.1093/oxfordjournals.cje.a035309, Cambridge Journal of Economics, 19(1), 5-24 (1995).

Geiger, S., y Finch, J., Making Incremental Innovation Tradable in Industrial Service Settings, https://doi.org/10.1016/j.jbusres.2016.02.015, Journal of Business Research, 69(7), 2463-2470 (2016).

Global Entrepreneurship Monitor (GEM), Global Report 2017/18, Global Entrepreneurship Research Association (2018).

Hofstede, G., y Hofstede, G.J., Cultures and Organizations: Software of the Mind, Revised and Expanded, $2^{\underline{a}}$ Ed., Nueva York, Estados Unidos (2005).

Khosravi, P., Newton, C., y Rezvani, A., Management Innovation: A Systematic Review and Meta-analysis of Past Decades of Research, https://doi.org/10.1016/j.emj.2019.03.003, European Management Journal, 37(6), 694-707 (2019).

Mardani, A., Nikoosokhan, S., y otros dos autores, The Relationship Between Knowledge Management and Innovation Performance, https://doi.org/10.1016/j.hitech.2018.04.002, Journal of High Technology Management Research, 29(1), 1226 (2018).

Lundvall, B., Johnson, B., y otros dos autores. National Systems of Production, Innovation and Competence Building, https://doi.org/10.1016/S0048-7333(01)00137-8, Research Policy, 31(2), 213-231 (2002).

Mihi, A., García, V.J., y Rojas, R.M., Knowledge Creation, Organization Learning and their Effects on Organizational Performance, http://dx.doi.org/10.5755/j01.ee.22.3.521, Inzinerine Ekonomika-Engineering Economics, 22(3), 309-318 (2011).

Nonaka, I., von Krogh, G., y Voelpel, S., Organizational Knowledge Creation Theory: Evolutionary Paths and Future Advances, https://doi.org/10.1177/0170840606066312, Organization Studies, 27(8), 1179-1208 (2006).

Nonaka, I., y von Krogh, G., Tacit Knowledge and Knowledge Conversion: Controversy and Advancement in Organizational Knowledge Creation Theory, https://doi.org/10.1287/orsc.1080.0412, Organization Science, 20(3), 635-652 (2009).

Nguyen, D.Q., The Impact of Intellectual Capital and Knowledge Flows on Incremental and Radical Innovation: Empirical Findings from a Transition Economy of Vietnam, https://doi.org/10.1108/APJBA-03-2018-0044, Asia-Pacific Journal of Business Administration, 10(2/3), 149-170 (2018).

Ode, E., y Ayavoo, R., The Mediating Role of Knowledge Application in the Relationship between Knowledge Management practices and firm innovation, https://doi.org/10.1016/j.jik.2019.08.002, Journal of Innovation \& Knowledge, 5(3), 210-218 (2019).

Quintane, E., Mitch R., y otros dos autores, Innovation as a Knowledge-based Outcome, https://doi.org/10.1108/13673271111179299, Journal of Knowledge Management, 15(6), 928-947 (2011).

Ringberg, T., Reihlen, M., y Rydén, P. The Technology-mindset Interactions: Leading to Incremental, Radical or Revolutionary Innovations, https://doi.org/10.1016/j.indmarman.2018.06.009, Industrial Marketing Management, 79, 102-113 (2019).

Serenko, A., y Dumay, J., Citation Classics Published in Knowledge Management Journals. Part I: articles and their characteristics, https://doi.org/10.1108/JKM-06-2014-0220, Journal of Knowledge Management, 19(2), $401-431$ (2015).

Scaringella, L., Knowledge, Knowledge Dynamics, and Innovation: Exploration of the Internationalization of a Multinational Corporation, https://doi.org/10.1108/EJIM-05-2015-0031, European Journal of Innovation Management, 19(3), 337-361(2016).

Subramaniam, M., y Youndt, M.A., The Influence of Intellectual Capital on the Types of Innovative Capabilities, https://doi.org/10.5465/amj.2005.17407911, Academy of Management Journal, 48(3), 450-463(2005).

Souto, J.E., Business Model Innovation and Business Concept Innovation as the Context of Incremental Innovation and Radical Innovation, https://doi.org/10.1016/j.tourman.2015.05.017, Tourism Management, 51, 142-155 (2015).

Tellis, G.J., Prabhu, J.C., y Chandy, R.K., Radical Innovation across Nations: The Preeminence of Corporate Culture, https://doi.org/10.1509/jmkg.73.1.003, Journal of Marketing, 73(1), 3-23 (2009).

Walecka-Jankowska, K., Relationship between Knowledge Management and Innovation, https://doi.org/10.5755/j01.ss.90.4.14260, Social Sciences, 90(4), 55-66 (2015). 